\title{
On stability of delay difference equations with variable coefficients: successive products tests
}

\section{Elena Braverman ${ }^{\text {* }}$ and Başak Karpuz ${ }^{2}$}

\section{"Correspondence:}

maelena@math.ucalgary.ca

${ }^{1}$ Department of Mathematics and

Statistics, University of Calgary, 2500

University Drive N. W., Calgary,

AB T2N 1N4, Canada

Full list of author information is

available at the end of the article

\begin{abstract}
In this paper, we report an error in the paper of the first author in Advances in Difference Equations, 2009, article 104310, present the revised versions of the theorem with several examples, and outline the cases when the previous result is valid.
\end{abstract}

\section{Introduction}

The purpose of this short note is to indicate an error in the previous paper [1] published in 'Advances in Difference Equations' and an inaccuracy in the recent paper [2]; to present a corrected result for [1] and clarification for [2]; and to outline the cases when the analogue of the result of [1] is still correct.

Consider the equation

$$
x(n+1)=\sum_{\ell=1}^{m} a_{\ell}(n) x\left(h_{\ell}(n)\right) \quad \text { for } n \geq n_{0},
$$

where $\left\{a_{\ell}(n)\right\}$ are sequences of real numbers, and $\left\{h_{\ell}(n)\right\}$ are sequences of integers such that there exists a nonnegative integer $\tau$ satisfying $n-\tau \leq h_{\ell}(n) \leq n$ for all $n \geq n_{0}$ and $\ell=1,2, \ldots, m$.

Theorem A Suppose that $n-h_{\ell}(n)<d$ for some $d \in \mathbb{N}, \ell=1,2, \ldots, m$, and there exists $r \in \mathbb{N}$ such that

$$
\limsup _{n \rightarrow \infty} \prod_{j=0}^{r} \sum_{\ell=1}^{m}\left|a_{\ell}(n-j)\right|<1 .
$$

Then (1) is exponentially stable.

Example 1 (Counterexample to Theorem A) Consider the delay difference equation

$$
x(n+1)=a(n) x(h(n)) \quad \text { for } n \geq 0,
$$


where

$$
a(n)=\left\{\begin{array}{ll}
p, & n=2 l, \\
q, & n=2 l+1
\end{array} \text { and } \quad h(n)= \begin{cases}n, & n=2 l, \\
n-1, & n=2 l+1\end{cases}\right.
$$

for some $p, q \in \mathbb{R}$. Simple computation gives us that the solution of (3) is

$$
x(n)= \begin{cases}q^{l} x(0), & n=2 l, \\ p q^{l} x(0), & n=2 l+1,\end{cases}
$$

which is stable if and only if $|q|<1$. More precisely, we have $\lim _{n \rightarrow \infty}|x(n)|=\infty$ for any $|q|>1$ provided that $x(0) \neq 0$. If we compute (2) with $r=1$ for (3), we get

$$
\limsup _{n \rightarrow \infty}|a(n) a(n-1)|=|p q|
$$

showing that the assumption of Theorem A is fulfilled if $|p q|<1$. However, we can find $p, q \in \mathbb{R}$ such that $|q| \geq 1$ and $|p q|<1$, for instance, $p=1 / 2$ and $q=6 / 5$. In this case, the right-hand side in (6) is $3 / 5<1$, but by (5) $x(2 l)=1.2^{l} x(0), x(2 l+1)=0.5 \cdot 1.2^{l}$, which is a divergent sequence, the solution is unstable.

Hence, in general, Theorem A is incorrect.

Let us note that in [2] and further in this paper, we apply the idea of reduction of higher (but bounded) order equations to first-order matrix equations. This method was widely used in [3-5] and in the earlier paper [6].

Also, in the discussion section of [2], the inequality

$$
\rho\left(A_{k(n)} A_{k(n)-1} \cdots A_{n}\right) \leq \lambda
$$

is considered as a sufficient asymptotic stability condition for the trivial solution of the first-order matrix equation

$$
X_{n+1}=A_{n} X_{n}
$$

Here $A_{n}$ are $d \times d$ matrices, $\rho(A)$ is the spectral radius of the matrix $A, \lambda \in(0,1), n_{0} \in \mathbb{N}$, and $k(n) \geq n$ is a certain number which exists for any $n \geq n_{0}$. Similarly, the condition

$$
\limsup _{n \rightarrow \infty} \rho\left(A_{n+k-1} A_{n+k-2} \cdots A_{n}\right)<1
$$

is treated as a sufficient exponential stability condition for the trivial solution. This is not true, as the example from [7, Example 4.17, pp.190-191] illustrates (here $k=1, k(n)=n$ ); see also the recent review [8] and Example 2 below.

Example 2 Equation (8), with

$$
A_{2 m}=\left(\begin{array}{cc}
0 & 1.2 \\
0.6 & 0
\end{array}\right), \quad A_{2 m+1}=\left(\begin{array}{cc}
0 & 0.6 \\
1.2 & 0
\end{array}\right), \quad m=0,1,2, \ldots
$$


satisfies $\rho\left(A_{n}\right)=0.6 \sqrt{2}<1$ since both $A_{2 m}$ and $A_{2 m+1}$ have eigenvalues $\pm 0.6 \sqrt{2}$. However, if we assume $X_{0}=(0,1)^{T}$, then simple calculations lead to $X_{2 m}=\left(0,1.44^{m}\right)^{T}$, thus the system is unstable.

On the other hand, if we use in Example 2 the norm

$$
\|A\|=\sup _{\|X\|=1}\|A X\|
$$

where $\|\cdot\|$ is the Euclidean vector norm, instead of the spectral radius, then $\left\|A_{m n}\right\|=$ $\left\|A_{2 m+1}\right\|=1.2>1$ since $\left\|A_{2 m}(0,1)^{T}\right\|=\left\|A_{2 m+1}(1,0)^{T}\right\|=1.2$, where $B^{T}$ is the transpose of $B$.

We will use the following result in the recovery of Theorem A, which was obtained in [9]; see also [10, 11].

Theorem B $\left(\left[9\right.\right.$, Theorem 2]) Let $m \in \mathbb{N}$ and $f: \mathbb{Z} \times \mathbb{R}^{m} \rightarrow \mathbb{R}$. If there exists $\lambda \in(0,1)$ such that

$$
\left|f\left(n, u_{1}, u_{2}, \ldots, u_{m}\right)\right| \leq \lambda \max _{1 \leq j \leq m}\left\{\left|u_{j}\right|\right\} \quad \text { for all } n \geq n_{0},
$$

then

$$
x_{n+1}=f\left(n, x_{n}, x_{n-1}, \ldots, x_{n-m+1}\right) \quad \text { for } n \geq n_{0}
$$

is globally exponentially stable. More precisely, any solution satisfies

$$
\left|x_{n}\right| \leq \lambda^{\left(n-n_{0}\right) / m} \max _{n_{0}-m+1 \leq j \leq n_{0}}\left\{\left|x_{j}\right|\right\} \quad \text { for all } n \geq n_{0} .
$$

\section{Main results}

For $k \in \mathbb{N}$, define a sequence

$$
b_{k}(n):= \begin{cases}1, & k=0, \\ \sum_{\ell=1}^{m}\left|a_{\ell}(n)\right| b_{k-1}\left(h_{\ell}(n)-1\right), & k \geq 1\end{cases}
$$

for $n \geq n_{0}+k(\tau+1)$.

Theorem 1 (Correction of Theorem A) Suppose that there exists $r \in \mathbb{N}$ such that

$$
\limsup _{n \rightarrow \infty} b_{r}(n)<1
$$

Then (1) is exponentially stable.

Proof Let us prove for all $k \in \mathbb{N}$ that

$$
|x(n+1)| \leq b_{k}(n) \max _{n-k(\tau+1) \leq j \leq n}\{|x(j)|\} \quad \text { for all } n \geq n_{0}+k(\tau+1) .
$$


We proceed by induction in $k$. From (1), for $k=1$, we have

$$
\begin{aligned}
|x(n+1)| & \leq \sum_{\ell=1}^{m}\left|a_{\ell}(n)\right|\left|x\left(h_{\ell}(n)\right)\right| \\
& \leq \sum_{\ell=1}^{m}\left|a_{\ell}(n)\right| \max _{n-(\tau+1) \leq j \leq n}\{|x(j)|\} \\
& =b_{1}(n) \max _{n-(\tau+1) \leq j \leq n}\{|x(j)|\}
\end{aligned}
$$

for all $n \geq n_{0}+\tau+1$. Thus, the claim is true for $k=1$. Assume now that the claim is true for some $k \geq 1$. From (12) and (13), for all $n \geq n_{0}+(k+1)(\tau+1)$, we have

$$
\begin{aligned}
|x(n+1)| & \leq \sum_{\ell=1}^{m}\left|a_{\ell}(n)\right| b_{k}\left(h_{\ell}(n)-1\right) \max _{h_{\ell}(n)-1-k(\tau+1) \leq j \leq h_{\ell}(n)-1}\{|x(j)|\} \\
& \leq \sum_{\ell=1}^{m}\left|a_{\ell}(n)\right| b_{k}\left(h_{\ell}(n)-1\right) \max _{n-(k+1)(\tau+1) \leq j \leq n}\{|x(j)|\} \\
& =b_{k+1}(n) \max _{n-(k+1)(\tau+1) \leq j \leq n}\{|x(j)|\},
\end{aligned}
$$

which shows that (12) is true when $k$ is replaced with $(k+1)$. Using (12) with $k=r$, we see that the solution is exponentially stable by Theorem $B$.

Theorem 1 with $r=1$ immediately yields the following result.

\section{Corollary 1 Assume that}

$$
\limsup _{n \rightarrow \infty} \sum_{\ell=1}^{m}\left|a_{\ell}(n)\right|<1
$$

Then (1) is exponentially stable.

Remark 1 The claim of Theorem A for $r=0$ is correct.

Setting $r=2$ in Theorem 1, we obtain the following corollary, which is also proved in [1, Theorem 2.17].

\section{Corollary 2 Assume that}

$$
\limsup _{n \rightarrow \infty} \sum_{\ell_{1}=1}^{m}\left|a_{\ell_{1}}(n)\right| \sum_{\ell_{2}=1}^{m}\left|a_{\ell_{2}}\left(h_{\ell_{1}}(n)-1\right)\right|<1 .
$$

Then (1) is exponentially stable.

Remark 2 Theorem A for the nondelay equation

$$
x(n+1)=a(n) x(n) \quad \text { for } n \geq n_{0}
$$


is correct. Indeed, Theorem 1 reduces to Theorem A since for $k \geq 1$, we get

$$
b_{k}(n)=\prod_{j=0}^{k-1}|a(n-j)| \quad \text { for } n \geq n_{0}+k .
$$

Setting $r=3$ in Theorem 1 gives us the following corollary.

\section{Corollary 3 Assume that}

$$
\limsup _{n \rightarrow \infty} \sum_{\ell_{1}=1}^{m}\left|a_{\ell_{1}}(n)\right| \sum_{\ell_{2}=1}^{m}\left|a_{\ell_{2}}\left(h_{\ell_{1}}(n)-1\right)\right| \sum_{\ell_{3}=1}^{m}\left|a_{\ell_{3}}\left(h_{\ell_{2}}\left(h_{\ell_{1}}(n)-1\right)-1\right)\right|<1 .
$$

Then (1) is exponentially stable.

Example 3 Consider the delay difference equation (3) with (4), where $p, q \in \mathbb{R}$, which can be written in the two equivalent forms:

$$
x(n+1)=-a_{1}(n) x(n)-a_{2}(n) x(n-1) \quad \text { for } n \geq 0
$$

and

$$
x(n+1)-x(n)=-\left(a_{1}(n)+1\right) x(n)-a_{2}(n) x(n-1) \quad \text { for } n \geq 0,
$$

where

$$
a_{1}(n)=\left\{\begin{array}{ll}
p, & n=2 l, \\
0, & n=2 l+1
\end{array} \text { and } \quad a_{2}(n)= \begin{cases}0, & n=2 l, \\
q, & n=2 l+1 .\end{cases}\right.
$$

Computing $\left\{b_{k}(n)\right\}$ defined by (11), we see that

$$
b_{k}(n)=\left\{\begin{array}{ll}
\left|p q^{k-1}\right|, & n=2 l, \\
\left|q^{k}\right|, & n=2 l+1
\end{array} \quad \text { for } k \in \mathbb{N}, n \geq 2 k .\right.
$$

Equation (3) is exponentially stable by Theorem 1 if $|q|<1$ because there always exists $r \in \mathbb{N}$ such that $\left|p q^{r-1}\right|<1$ and $\left|q^{r}\right|<1$. From (5), we see that $|q|<1$ is the best possible condition for the global exponential stability of (3) with (4).

Application of a recent result [12, Theorem 6] to (14) gives us $(1+|p|)|q|<1$, which implies $|q|<1$.

The so-called '3/2-test' (see [13] and [1, Theorem A]) can be applied to (15) if $p>-1$ and $q>0$, and ensures global exponential stability when

$$
p+q+2<\frac{3}{2}+\frac{1}{2 \cdot 2}=\frac{7}{4} \quad \text { or equivalently } \quad p+q<-\frac{1}{4}
$$

for which $0<q<3 / 4$ is necessary.

It is obvious that these two results and Corollary 1 cannot deliver any answer for the exponential stability when $p=1$ and $q=1 / 2$. 
As mentioned in Remark 2, Theorem A is valid for a nondelay scalar equation. Next, any higher-order (of the order not exceeding $d$ ) equation (1), with $n-d<h_{\ell}(n) \leq n$, can be rewritten as the first-order system

$$
X(n+1)=A(n) X(n), \quad n=0,1,2, \ldots,
$$

where $X(n) \in \mathbb{R}^{d}, A(n)$ are $d \times d$ matrices. Indeed, denote $X(0)=(x(-d+1), x(-d+$ $2), \ldots, x(0))^{T}, X(n)=(x(n d-d+1), x(n d-d+2), \ldots, x(n d))^{T}$ and rewrite (1) as

$$
x(n)=\sum_{j=1}^{d} b(n, j) x(n-j), \quad n \in \mathbb{N},
$$

where

$$
b(n, j)=\sum_{l \in\left\{1, \ldots, m \mid h_{l}(n)=n-j\right\}} a_{l}(n) .
$$

Then we can define the matrix $A(0)=(c(i, j))_{i=1}^{d}$ as follows:

$$
\begin{aligned}
& x(1)=\sum_{j=1}^{d} b(1, j) x(1-j)=\sum_{j=1}^{d} c(1, j) x(1-j), \\
& x(2)=\sum_{j=1}^{d} b(2, j) x(2-j)=\sum_{j=2}^{d} b(2, j) x(2-j)+b(2,1) \sum_{j=1}^{d} b(1, j) x(1-j) \\
& =\sum_{j=1}^{d-1} b(2, j+1) x(1-j)+\sum_{j=1}^{d} b(2,1) b(1, j) x(1-j)=\sum_{j=1}^{d} c(2, j) x(1-j), \\
& x(d)=\sum_{j=1}^{d} c(d, j) x(1-j),
\end{aligned}
$$

and $X(1)=A(0) X(0)$. Similarly, we construct $A(n), n \in \mathbb{N}$ and obtain system (16). Since $\|X(n)\| \geq|x(j)|, j=n d-d+1, n d-d+2, \ldots, n d$, exponential (asymptotical) stability of (16) implies the relevant stability of (1). We recall that (16) is exponentially stable if there exist $n_{0} \in \mathbb{N}, L>0$, and $\mu \in(0,1)$ such that $\|X(n)\| \leq L \mu^{n}\|X(0)\|, n \geq n_{0}$.

Theorem 2 If there exist $\lambda \in(0,1), M>0$, and $n_{0} \in \mathbb{N}$ such that $\|A(n)\| \leq M$ and $\left\|\prod_{j=n}^{n+k-1} A(j)\right\| \leq \lambda$ for every $n \geq n_{0}$ and for some positive integer $k$, then (16) is exponentially stable.

Proof Without loss of generality, we can assume $M>1$ and $\prod_{j=0}^{n_{0}-1}\|A(j)\| \leq M$. Further, for any $n \geq n_{0}$ denote $m=\left[\frac{n-n_{0}-1}{k}\right]$, where $[t]$ is the integer part of $t$, and obtain the estimate

$$
\begin{aligned}
\|X(n)\| & =\|A(n-1) \cdots A(0) X(0)\| \leq\|A(n-1) \cdots A(0)\|\|X(0)\| \\
& =\left\|\prod_{j=n_{0}+k m}^{n-1} A(j) \cdots \prod_{j=n_{0}}^{n_{0}+k-1} A(j) \prod_{j=0}^{n_{0}-1} A(j)\right\|\|X(0)\|
\end{aligned}
$$




$$
\begin{aligned}
& \leq\left\|\prod_{j=n_{0}+k m}^{n-1} A(j)\right\| \cdots\left\|\prod_{j=n_{0}}^{n_{0}+k-1} A(j)\right\|\left\|\prod_{j=0}^{n_{0}-1} A(j)\right\|\|X(0)\| \\
& \leq M \lambda^{m} M^{k} \leq M^{k+1}\left(\frac{1}{\lambda}\right)^{1+n_{0} / k}\left(\lambda^{1 / k}\right)^{n}\|X(0)\|=L \mu^{n}\|X(0)\|
\end{aligned}
$$

for $n \geq n_{0}$, where $L=M^{k+1} \lambda^{-1-n_{0} / k}, \mu=\lambda^{1 / k}$.

Example 4 (Example 6 in [2]) If in (16)

$$
A_{2 m}=\frac{1}{2}\left(\begin{array}{ll}
5.1 & 4.9 \\
4.9 & 5.1
\end{array}\right), \quad A_{2 m+1}=\frac{1}{2}\left(\begin{array}{cc}
7.1 & -6.9 \\
-6.9 & 7.1
\end{array}\right), \quad m=0,1,2, \ldots
$$

then both $A_{2 m}$ and $A_{2 m+1}$ have the norms exceeding one (they have eigenvalues of 5 and 7 , respectively), but the product

$$
A_{2 m} A_{2 m+1}=A_{2 m+1} A_{2 m}=\left(\begin{array}{cc}
0.6 & -0.1 \\
-0.1 & 0.6
\end{array}\right)
$$

has the norm $\left\|A_{2 m} A_{2 m+1}\right\|=\left\|A_{2 m+1} A_{2 m}\right\|=0.7<1$, thus (16) is exponentially stable.

Example 5 Consider (16) with a 4-periodic matrix $A(n)$, where $A_{4 m+j}=\left(\begin{array}{cc}0 & -1.1 \\ 1.1 & 0\end{array}\right), m=$ $0,1,2, \ldots, j=1,2,3, A_{4 m}=\left(\begin{array}{cc}0 & -0.7 \\ 0.7 & 0\end{array}\right)$. Then $\left\|A_{4 m+j}\right\|=1.1>1, j=1,2,3$, but (16) is exponentially stable since $A_{n+3} A_{n+2} A_{n+1} A_{n}=\left(\begin{array}{cc}0.9317 & 0 \\ 0 & 0.9317\end{array}\right)$ for any $n=0,1,2, \ldots$, and $\lambda=$ $\left\|A_{n+3} A_{n+2} A_{n+1} A_{n}\right\|=0.9317<1$.

\section{Discussion}

The dynamics of higher-order difference equations with variable coefficients, as well as of non-autonomous systems of difference equations, is much more complicated than that of the relevant autonomous models; see, for example, $[8,14]$. For example, the fact that the spectral radius of each matrix is less than one does not imply exponential stability of the system. On the other hand, as demonstrated in Example 5, non-autonomous systems, where some matrices have norms exceeding one, can still be exponentially stable. The challenge is to extend recursive results to other type of stability, for example, asymptotic and $l^{p}$ stability; see, for example, [3].

Regarding generalizations to some types of nonlinear models, the analogue of Theorem 1 can be found in [2], while Theorem 2 can be reformulated for the nonlinear first-order system

$$
X(n+1)=F_{n}(X(n)), \quad n=0,1,2, \ldots,
$$

in the following way, with the same proof repeated.

Theorem 3 If there exist $\lambda \in(0,1), k \in \mathbb{N}, M>0$, and $n_{0} \in \mathbb{N}$ such that $\left\|F_{n}(X)\right\| \leq M\|X\|$ and $\left\|F_{n+k-1}\left(\cdots F_{n+1}\left(F_{n}(X)\right) \cdots\right)\right\| \leq \lambda\|X\|$ for any $X \in \mathbb{R}^{d}$ and $n \geq n_{0}$, then (18) is uniformly exponentially stable. 
Again, the case of possible asymptotic stability when

$$
\left\|F_{n+k-1}\left(\cdots F_{n+1}\left(F_{n}(X)\right) \cdots\right)\right\| \leq \lambda_{n}\|X\|
$$

and $\lim \sup _{n \rightarrow \infty} \lambda_{n}=1$ is still to be considered.

\section{Competing interests}

The authors declare that they have no competing interests.

\section{Authors' contributions}

All authors contributed equally to the manuscript and read and approved the final draft.

\section{Author details}

'Department of Mathematics and Statistics, University of Calgary, 2500 University Drive N. W., Calgary, AB T2N 1N4, Canada. ${ }^{2}$ Department of Mathematics, Faculty of Science and Arts, Afyon Kocatepe University, ANS Campus, Afyonkarahisar, 03200, Turkey.

\section{Acknowledgements}

The authors are grateful to the anonymous reviewer whose valuable comments greatly contributed to the presentation of the paper. EB was partially supported by the NSERC Research grant. This work was partially completed while BK was on one-year leave at the Department of Mathematics and Statistics, University of Calgary, Canada, in the framework of Doctoral Research Scholarship of the Council of Higher Education of Turkey. The authors are also grateful to the University of Calgary who supported this publication.

Received: 7 June 2012 Accepted: 24 September 2012 Published: 9 October 2012

\section{References}

1. Berezansky, L, Braverman, E: Exponential stability of difference equations with several delays: recursive approach. Adv. Differ. Equ. 2009, Article ID 104310 (2009)

2. Braverman, E, Karpuz, B: On global asymptotic stability of nonlinear higher-order difference equations. J. Comput. Appl. Math. 236, 2803-2812 (2012)

3. Győri, I, Horváth, L: A new view of the PP-theory for a system of higher order difference equations. Comput. Math. Appl. 59, 2918-2932 (2010)

4. Győri, I, Horváth, L: Asymptotic constancy in linear difference equations: limit formulae and sharp conditions. Adv. Differ. Equ. 2010, Article ID 789302 (2010)

5. Győri, I, Horváth, L: Asymptotic behaviour of the solutions of a nonautonomous linear delay difference system. Appl. Math. Comput. 217, 4205-4216 (2010)

6. Berezansky, L, Braverman, E: On exponential dichotomy for linear difference equations with bounded and unbounded delay. In: Differential and Difference Equations and Applications, pp. 169-178. Hindawi Publ., New York (2006)

7. Elaydi, S: An Introduction to Difference Equations. Undergraduate Texts in Mathematics. Springer, New York (2005)

8. Pötzsche, C: Bifurcations in nonautonomous dynamical systems: results and tools in discrete time. In: Liz, E, Mañosa, V (eds.) Proceedings of the Workshop 'Future Directions in Difference Equations', Vigo (Spain), June 13-17, 2011, vol. 69, pp. 163-212. Servizo de Publicacións da Universidade de Vigo, Vigo (2011). ISBN:978-84-8158-541-4

9. Liz, E, Ferreiro, JB: A note on the global stability of generalized difference equations. Appl. Math. Lett. 15, 655-659 (2002)

10. Berezansky, L, Braverman, E, Liz, E: Sufficient conditions for the global stability of nonautonomous higher order difference equations. J. Differ. Equ. Appl. 11, 785-798 (2005)

11. Stević, S: Behavior of the positive solutions of the generalized Beddington-Holt equation. Panam. Math. J. 10, 77-85 (2000)

12. El-Morshedy, HA: New explicit global asymptotic stability criteria for higher order difference equations. J. Math. Anal. Appl. 336, 262-276 (2007)

13. Erbe, LH, Xia, H, Yu, JS: Global stability of a linear nonautonomous delay difference equation. J. Differ. Equ. Appl. 1, 151-161 (1995)

14. Győri, I, Horváth, L: P ${ }^{P}$-solutions and stability analysis of difference equations using the Kummer's test. Appl. Math. Comput. 217, 10129-10145 (2011)

doi:10.1186/1687-1847-2012-177

Cite this article as: Braverman and Karpuz: On stability of delay difference equations with variable coefficients: successive products tests. Advances in Difference Equations 2012 2012:177. 